Int. J. Electrochem. Sci., 11 (2016) 5427 - 5440

International Journal of

ELECTROCHEMICAL

SCIENCE

www.electrochemsci.org

\title{
Application of Single-Walled Carbon Nanotubes/Au Nanosol Modified Electrode for the Electrochemical Determination of Esculetin in Cortex Fraxini
}

Yuanyuan Yao ${ }^{1,2}$, Xiaomei Zhang ${ }^{1}, \mathrm{Na} \mathrm{Li}^{1}$, Xuming Liang ${ }^{1}$, Yangping Wen ${ }^{2,3}$, Hui Zhang ${ }^{2,3}$, Yilong Chen , Dajian Yang ${ }^{1, *}$, and Jingkun $\mathrm{Xu}^{2, *}$

${ }^{1}$ Chongqing Academy of Chinese Materia Medica, Chongqing 400065, P. R. China

${ }^{2}$ School of Pharmacy, Jiangxi Science and Technology Normal University, Nanchang 330013, PR China

${ }^{3}$ Key Laboratory of Applied Chemistry, Jiangxi Agricultural University, Nanchang 330045, PR China

*E-mail: yangdajian@ foxmail.com, xujingkun@tsinghua.org.cn

doi: $10.20964 / 2016.07 .59$

Received: 15 April 2016 / Accepted: 19 May 2016 / Published: 4 June 2016

A novel simple, sensitive and selective electrochemical sensor was successfully prepared for the determination of esculetin in Cortex Fraxini based on the carboxylic acid-functionalized single-walled carbon nanotubes-Nafion-Au nanosol nanocomposite modified glassy carbon electrode (c-SWCNTs$\mathrm{NF}-\mathrm{AuNs} / \mathrm{GCE}$ ). Scanning electron microscopy, energy dispersive X-ray spectroscopy, electrochemical impedance spectroscopy and cyclic voltammetry were carried out to characterize the properties of c-SWCNTs-NF-AuNs nanocomposite. Owing to the synergistic effects of large surface area, superior electrical conductivity, and large amount of chemically active sites of c-SWCNTs, together with the good biocompatibility and high conductivity of AuNs, the c-SWCNTs-NFAuNs/GCE exhibited a good electrocatalytic activity to esculetin with wide linear range of 0.004-55 $\mu \mathrm{M}$ and low detection limit of $0.12 \mathrm{nM}$. Additionally, the modified electrode was employed for analysis of esculetin in Cortex Fraxini with satisfactory results.

Keywords: Electrochemical determination; Differential pulse voltammetry; Single-walled carbon nanotube; Au nanosol; Esculetin;

\section{$\underline{\text { FULL TEXT }}$}

(C) 2016 The Authors. Published by ESG (www.electrochemsci.org). This article is an open access article distributed under the terms and conditions of the Creative Commons Attribution license (http://creativecommons.org/licenses/by/4.0/). 